\title{
Both experimental hypothyroidism and hyperthyroidism increase cardiac irisin levels in rats
}

\author{
Atici $\mathrm{E}^{1}$, Menevse $\mathrm{E}^{2}$, Baltaci $\mathrm{AK}^{3}$, Mogulkoc $\mathrm{R}^{3}$ \\ Baskent University, Vocational School of Health, Department of Therapy \& Rehabilitation, Ankara, Turkey. \\ rasimmogulkoc@yahoo.com
}

\begin{abstract}
Irisin is a newly discovered myokine and adipokine that increases total body energy expenditure. The aim of this study was to determine the effect of experimental hypothyroidism and hyperthyroidism on the levels of irisin in heart tissue in rats. The study was performed on the 40 male Sprague-Dawley rats. Experimental groups were designed as; Control, Hypothyroidism, Hypothyroidism+L-Thyroxine, Hyperthyroidism and Hyperthyroidism + PTU. Following 3 weeks experimental period, irisin levels were determined in heart tissues. Hypothyroidism group values of irisin were higher than in the control group, but lower than in the hyperthyroidism group. The hyperthyroidism group had the highest levels of cardiac irisin. The results of the study showed that the experimental hypothyroidism and hyperthyroidism increased the heart irisin levels, but the increase in the hyperthyroidism group was much higher than in the hypothyroidism group. However, treatment of hypothyroidism and hyperthyroidism corrected cardiac irisin levels (Fig. 1, Ref. 28). Text in PDF www.elis.sk.

KEY WORDS: hyperthyroidism, hypothyroidism, heart tissue, irisin, rat.
\end{abstract}

\section{Introduction}

Irisin is myokine and it has been first time expressed in the skeletal muscle. As a result of researches, it is possible to synthesize in many tissues (skeletal muscle, fat tissue, cardiac tissue, intracranial arteries, kidneys, myelin sheath, neural cells, optic nerve, Overlap, Purkinje cells, rectum, salivary glands, juvenile sweat glands, stomach, testes) and it is indicated that the main source is skeletal muscle and fat tissue $(1,2)$. Irisin is an exercise protein, which is released to circulation. Irisin is a peptide hormone that divides the plasma membrane protein fibronectin type III domain containing 5 (FNDC5). This hormone access the white and brown fat tissue by circulation. Irisin lead to energy expenditure this way (3). FNDC5 is a precursor of irisin and overexpression of this precursor increases the use of oxygen and heat production. Irisin is an active lipolysis and energy expenditure agent. (4). It has been also suggested that serum irisin and cardiovascular diseases might have a relationship $(5,6,7,8,9)$. It has been reported that heart muscle had a high FNDC expression and production of irisin than skeletal muscle (10). Heart tissue irisin levels are reduced after the myocardial infarction and might be used as diagnostic agent. (11). It has been also suggested that serum irisin levels might be used as biomarkers

${ }^{1}$ Baskent University, Vocational School of Health, Department of Therapy \& Rehabilitation, Ankara, Turkey, ${ }^{2}$ Selcuk University, Faculty of Medicine, Department of Biochemistry, Konya, Turkey, and ${ }^{3}$ Selcuk University, Faculty of Medicine, Department of Physiology, Konya, Turkey

Address for correspondence: R. Mogulkoc, Selcuk University, Medical Faculty, Department of Physiology, 42075 Selcuklu, Konya, Turkey. Phone: +90.3322443857 , Fax: +90.3322412184

Acknowledgement: This study was supported by a grant Selcuk University, Scientific Research Council (Grant number is 14202034). for heart failure (3). Xie et al (12) determined that recombinant irisin has physiological effects on cardiomyoblast and myocardium.

Thyroid dysfunction is a risk factor for progression of cardiovascular diseases $(13,14,15)$. However, there are no long-term studies showing that patients with heart failure mediated by thyroid dysfunction have altered the incidence or prognosis (16). Thyroid hormones play an important role in the regulation of basal metabolic rate and in thermogenesis, which are likely to be affected by irisin. Or, conversely, irisin may be affecting a thyroid function. Thyroid hormones signal through mechanisms that affect energy consumption by central and peripheral pathways (17).

The aim of the study was to determine heart tissue irisin levels in experimental hypothyroidism and hyperthyroidism in rats.

\section{Material and methods}

\section{Experimental animals and experiment protocol}

An application was made to the Ethical Committee for Experimental Research on Animals of Baskent University's School of Medicine for the evaluation of the conformity of the study with ethical principles, and the approval of the committee was received by the document number DA/14 27. Based on the biostatistical pre-evaluation, it was decided to include 40 rats in the study with 8 rats in each study group.

The study included male Sprague-Dawley rats weighing between 250 and $300 \mathrm{gr}$ and supplied by the Baskent University's Experimental Animal Breeding and Research Centre. All procedures were planned in consideration of the "Guide for the Care and Use of Laboratory Animals". After the subjects were let to adapt to the laboratory conditions $\left(22 \pm 2{ }^{\circ} \mathrm{C}, 12\right.$ hours light and 
12 hours dark) for 2 weeks, they were divided into groups according to the experiment protocol. Hormone analyses in the study were conducted at the Physiology Laboratory of Selcuk University School of Medicine.

\section{Experiment groups}

The rats were fed on a standard diet in a light- and heat-controlled environment, and all four groups except the control group were supplemented with thyroid hormones for 3 weeks.

Group $1(\mathrm{n}=8)$ : Control: The rats in this group were sacrificed without being subjected to any procedure and the plasma obtained from their blood samples was stored at $-80{ }^{\circ} \mathrm{C}$ until the time of analysis.

Group $2(\mathrm{n}=8)$ : 6-n-propyl-2-thiouracil (PTU): To induce hypothyroidism, the rats in this group were administered 6-npropyl-2-thiouracil $(10 \mathrm{mg} / \mathrm{kg} /$ day $)$ by the intraperitoneal route daily for 3 weeks (10).

Group $3(\mathrm{n}=8)$ PTU + L-thyroxin: After hypothyroidism was induced by 2 -week PTU administration, the animals were administered high-dose L-thyroxin $(1.5 \mathrm{mg} / \mathrm{kg} /$ day) for 1 week.

Group $4(n=8)$ L-thyroxin: To induce hyperthyroidism, the rats were injected with $0.3 \mathrm{mg} / \mathrm{kg} /$ day of L-thyroxine through the intraperitoneal route for 3 weeks.

Group $5(\mathrm{n}=8)$ L-thyroxin + PTU: After hyperthyroidism was induced by 2 -week thyroxin injection, the animals were supplemented with $10 \mathrm{mg} / \mathrm{kg} /$ day PTU for one week.

At the end of three weeks, the rats were anesthetized (Ketamine $100 \mathrm{mg} / \mathrm{kg}$, chlorpromazine $10 \mathrm{mg} / \mathrm{kg}$; ip), and after blood samples were collected from their aorta, they were sacrificed. The samples were centrifuged at $4000 \mathrm{rpm}$ for 10 minutes to obtain plasma samples, which were then kept at $-80{ }^{\circ} \mathrm{C}$ until the time of analysis. The samples were used to measure heart tissue irisin levels utilizing relevant kits.

\section{Irisin analysis}

To determine irisin concentrations, Phoenix Pharmaceuticals' irisin (human, rat, mouse, rodent) ELISA kits (Catalogue

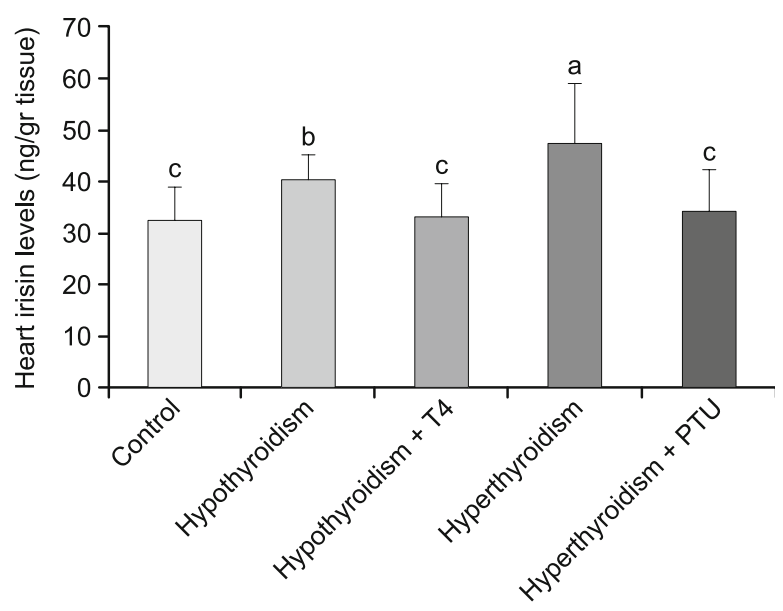

Fig. 1. Heart Irisin levels (ng/g tissue).
No: EK-067-29) were used. Concentrations of the hormone were shown as $\mathrm{ng} / \mathrm{ml}$.

Heart tissue ventricles were homogenised in phosphate buffer (Ph 7.4) by Misonix's Microscan ultrasonic homogenizer. After centrifugation at $3000 \mathrm{rpm}$ for 15 minutes, the upper supernatant was evaluated to the irisin analysis. Levels were given as ng/gr tissue.

\section{Statistical analysis}

SPSS statistics software was used for the statistical analysis. The results were described as mean \pm standard deviation. Kruskal-Wallis variance analysis was used in the comparison between groups and Mann-Whitney U test was employed for the value of $\mathrm{p}<0.05$, which was accepted as statistically significant.

\section{Results}

Irisin levels of the experimental groups were $32.50 \pm 6.55$; $40.53 \pm 4.69 ; 33.31 \pm 6.33 ; 47.52 \pm 11.70 ; 34.13 \pm 08.07 \mathrm{ng} / \mathrm{gr}$ respectively for groups 1, 2, 3, 4 and 5. Heart tissue irisin levels were different in the experimental groups. The highest irisin was determined in the hyperthyroidism groups $(\mathrm{p}<0.01)$. Hypothyroidism group had a high irisin level compared to other groups $(\mathrm{p}<0.01)$, but lower than in the hyperthyroidism group. However, treatment of thyroid dysfunction (groups 3 and 5) corrected deteriorated irisin levels and these levels were similar to the control group (Fig. 1).

\section{Discussion}

The main findings of our research suggested that both hypothyroidism and hyperthyroidism increased heart tissue irisin levels in rats. It has been seen that no research that investigated relation of heart irisin levels and thyroid hormones was done. Previously, few researches were present that determined heart irisin levels in different condition, such as doxorubicin treatment or exercise (4, 10). Aydin et al (4) investigated the presence of irisin in cardiac muscle, skeletal muscle, liver, kidney, peripheral nerve sheath, skin tissue and serum of rats age 12 and 24 months after the exercise. Young and old rats, who did not exercise, were observed to have no irisin in the skeleton; however, it had been released after an exercise. Serum irisin levels were reported to be higher in young rats that were exercising than in exercise-elderly. The presence of the main source of the irisin was also reported in other examined tissues, not only in the skeleton. Kuloglu et al (11) investigated the level of irisin in myocardial infarction (MI). The synthesis of the irisin hormone was observed to decrease after one to four hours from MI. It was thought that this decrease could be used as a diagnostic marker. They reported that the salivary and serum irisin hormone levels of acute MI patients fell within 48 hours and therefore they suggested that salivary and serum irisin levels could be used as biomarkers (9). Ates et al (18) investigated the effects of irisin hormone on thyroid tissue, fat tissue and metabolism of obese and thyroid patients as well. This study was conducted with 37 healthy and 37 Hashimoto disease-diagnosed adults, who were 
not yet treated. Compared to the control group, it was reported that the hypothyroid group had higher levels of irisin hormone, and the irisin level was negatively correlated with an increasing age in both the patient and healthy group. In all subjects, irisin levels were found to be positive with thyroid stimulating hormone (TSH) and negative with free T4.

In our study, heart irisin levels were found increased by about $25 \%$ in hypothyroidism group compared to the control. In their study involving 37 recently diagnosed Hashimoto's thyroiditis patients and 37 healthy volunteers, Ates et al (18) reported that irisin levels were elevated in hypothyroidism. This increase might have resulted from the structural impairment of the thyroid gland. In fact, Huh et al (19) demonstrated that FNDC5, an irisin precursor, was present in the thyroid gland and was destroyed in the case of inflammation of the thyroid gland, and that blood levels of irisin might increase after this destruction. In our study, the change in the thyroid gland was not examined from a structural perspective. However, in a previous study using the same experimental model, it was shown that PTU caused a structural impairment in the thyroid tissue (20). Samy et al (21) found elevated irisin levels in hypothyroidism, as we did in our study, and claimed that this increase was associated with oxidative stress and muscle injury. Thus, this may be another way of explaining the elevated irisin levels in experimental hypothyroidism. In the present study, the oxidative status was not evaluated in heart tissue. However, increased oxidative stress was reported in thyroid function impairment in previous studies of a similar nature $(22,23)$. Although these studies suggest that there may be a relation between the thyroid gland and irisin, there are others, which report that there is no relation between the two hormones $(24,25)$. In our research, irisin levels displayed significant increases especially in the case of hypothyroidism. It was reported in previous studies that the thyroid hormone $\mathrm{T} 3$ elevated blood irisin levels by increasing the expression of the first precursor of irisin, PGC-1 $\alpha$ (26).

Thyroid hormones play key roles in the regulation of the basal metabolic rate and thermogenesis, which are possibly affected by irisin. Thus, thyroid hormones may be directly or indirectly related to irisin or vice versa, with irisin affecting the thyroid function. In a previous study, plasma irisin levels were reported to be associated with thyroid hormones not in the basal condition, but due to muscle injury (27).

In the experimental studies, no relation was observed between irisin and thyroid hormones. However, the subjects in both studies had normal thyroid functions $(24,25)$. Conversely, in their study involving recently diagnosed hyperthyroidism and hypothyroidism patients, Ruchala et al (28) demonstrated that hypothyroid patients had lower irisin levels than the patients with hyperthyroidism. Additionally, it was found that irisin levels were correlated positively with free thyroxin levels and negatively with CK levels (28). Samy et al (21) showed that irisin levels varied depending on the trio metabolic status in thyroid models. Elevated heart irisin levels we found in hyperthyroidism in our study were similar to the results reported above.

It was established that the irisin hormone increased in heart tissue of experimental hypothyroidism and hyperthyroidism, and that this increase was more marked in hyperthyroidism. However, treatment of hypothyroidism and hyperthyroidism restored changed heart tissue irisin levels in rats.

\section{References}

1. Aydin S, Kuloglu T, Aydin S, Eren MN, Celik A, Yilmaz M, Kalayci M, Sahin I, Gungor O, Gurel A, Ogeturk M, Dabak O. Cardiac, skeletal muscle and serum irisin responses to with or without water exercise in young and old male rats: cardiac muscle produces more irisin than skeletal muscle. Peptides 2014; 52: 68-73.

2. Hofmann T, Elbelt U, Stengel A. Irisin as a muscle-derived hormone stimulating thermogenesis-a critical update. Peptides 2014; 54: 89-100.

3. Shen S, Gao R, Bei Y, Li J, Zhang H, Zhou Y, Yao W, Xu D, Zhou F, Jin M, Wei S, Wang K, Xu X, Li Y, Xiao J, Li X. Serum Irisin Predicts Mortality Risk in Acute Heart Failure Patients. Cell Physiol Biochem. 2017; 42 (2): 615-622.

4. Xiong XQ, Chen D, Sun HJ, Ding L, Wang JJ, Chen Q, Li YH, Zhou YB, Han Y, Zhang F, Gao XY, Kang YM, Zhu GQ. FNDC5 overexpression and irisin ameliorate glucose/lipid metabolic derangements and enhance lipolysis in obesity. Biochim Biophys Acta 2015; 1852 (9): $1867-1875$.

5. Gouveia MC, Vella JP, Cafeo FR, Affonso Fonseca FL, Bacci MR. Association between irisin and major chronic diseases: a review. Eur Rev Med Pharmacol Sci 2016; 20: 4072-4077.

6. Icli A, Cure E, Cumhur Cure M, Uslu AU, Balta S, Arslan S, Sakiz D, Kucuk A. Novel myokine: irisin may be an independent predictor for subclinic atherosclerosis in Behçet's disease. J Investig Med 2016; 64 : 875-881.

7. Deng W. Association of serum irisin concentrations with presence and severity of coronary artery disease. Med Sci Monit 2016; 22: 4193-4197.

8. Aronis KN, Moreno M, Polyzos SA, Moreno-Navarrete JM, Ricart W, Delgado E, de la Hera J, Sahin-Efe A, Chamberland JP, Berman R, Spiro A 3rd, Vokonas P, Fernández-Real JM, Mantzoros CS. Circulating irisin levels and coronary heart disease: association with future acute coronary syndrome and major adverse cardiovascular events. Int J Obes (Lond) 2015; 39: 156-161.

9. Aydin S, Aydin S, Kobat MA, Kalayci M, Eren MN, Yilmaz M, Kuloglu T, Gul E, Secen O, Alatas OD, Baydas A. Decreased saliva/serum irisin concentrations in the acute myocardial infarction promising for being a new candidate biomarker for diagnosis of this pathology. Peptides 2014; 56: 141-145.

10. Aydin S, Eren MN, Kuloglu T, Aydin S, Yilmaz M, Gul E, Kalayci M, Yel Y, Cakmak T. Alteration of serum and cardiac tissue adropin, copeptin, irisin and TRPM2 expressions in DOX treated male rats. Biotech Histochem 2015; 90 (3): 197-205.

11. Kuloglu T, Aydin S, Eren MN, Yilmaz M, Sahin I, Kalayci M, Sarman E, Kaya N, Yilmaz OF, Turk A, Aydin Y, Yalcin MH, Uras N, Gurel A, Ilhan S, Gul E, Aydin S. Irisin: a potentially candidate marker for myocardial infarction. Peptides 2014; 55: 85-91.

12. Xie C, Zhang Y, Tran TD, Wang H, Li S, George EV, Zhuang H, Zhang P, Kandel A, Lai Y, Tang D, Reeves WH, Cheng H, Ding Y, Yang LJ. Irisin controls growth, intracellular $\mathrm{Ca} 2+$ signals and mitokondrial thermogenesis in cardiomyoblast. Plos one 2015; 10 (8): e0136816.

13. Selmer C, Olesen JB, Hansen ML, von Kappelgaard LM, Madsen JC, Hansen PR, Pedersen OD, Faber J, Torp-Pedersen C, Gislason GH. 
Subclinical and overt thyroid dysfunction and risk of all-cause mortality and cardiovascular events: a large population study. J Clin Endocrinol Metab 2014; 99: 2372-2382.

14. Nanchen D, Gussekloo J, Westendorp RG, Stott DJ, Jukema JW, Trompet S, Ford I, Welsh P, Sattar N, Macfarlane PW, Mooijaart SP, Rodondi N, de Craen AJ. Subclinical thyroid dysfunction and the risk of heart failure in older persons at high cardiovascular risk. J Clin Endocrinol Metab 2012; 97: 852-861.

15. Gencer B, Collet TH, Virgini V, Bauer DC, Gussekloo J, Cappola AR, Nanchen D, den Elzen WP, Balmer P, Luben RN, Iacoviello M, Triggiani V, Cornuz J, Newman AB, Khaw KT, Jukema JW, Westendorp RG, Vittinghoff E, Aujesky D, Rodondi N. Subclinical thyroid dysfunction and the risk of heart failure events: an individual participant data analysis from 6 prospective cohorts. Circulation 2012; 126: 1040-1049.

16. Vargas-Uricoechea H, Bonelo-Perdomo A. Thyroid Dysfunction and Heart Failure: Mechanisms and Associations. Curr Heart Fail Rep. 2017; 14 (1): 48-58.

17. McAninch EA, Bianco AC. Thyroid hormone signaling in energy homeostasis and energy metabolism. Ann N Y Acad Sci. 2014; 1311: 77-87.

18. Ates I, Altay M, Topcuoglu C, Yılmaz FM. Circulating levels of irisin is elevated in hypothyroidism, a case-control study. Arch Endocrinol Metab 2015; 1-6.

19. Huh JY, Panagiotou G, Mougios V, Brinkoetter M, Vamvini MT, Schneider BE, Mantzoros CS. FNDC5 and irisin in humans: I. Predictors of circulating concentrations in serum and plasma and II. mRNA expression and circulating concentrations in response to weight loss and exercise. Metab Clin Exp 2012; 61: 1725-1738.

20. Mogulkoc R, Baltaci AK, Aydin L, Oztekin E, Sivrikaya A. The effect of thyroxin administration on lipid peroxidation in different tissues of rats with hypothyroidism. Acta Physiol Hung 2005; 92 (1): 39-46.
21. Samy DM, Ismail CA, Nassra RA. Circulating irisin concentrations in rat models of thyroid dysfunction - effect of exercise. Metabolism 2015; 64 (7): 804-813.

22. Mogulkoc R, Baltaci AK, Aydin L, Oztekin E, Tuncer I. Pinealectomy increases oxidant damage in kidney and testis caused by hyperthyroidism in rats. Cell Biochem Funct 2006; 24 (5): 449-453.

23. Mogulkoc R, Baltaci AK, Oztekin E, Aydin L, Sivrikaya A. Melatonin prevents oxidant damage in various tissues of rats with hyperthyroidism. Life Sci 2006; 79 (3): 311-315.

24. Ellefsen S, Vikmoen O, Slettaløkken G, Whist JE, Nygaard H, Hollan I, Rauk I, Vegge G, Strand TA, Raastad T, Rønnestad BR. Irisin and FNDC5: effects of 12-week strength training, and relations to muscle phenotype and body mass composition in untrained women. Eur J Appl Physiol 2014: 114; 1875-1888.

25. Stengel A, Hofmann T, Goebel-Stengel M, Elbelt U, Kobelt P, Klapp BF. Circulating levels of irisin in patients with anorexia nervosa and different stages of obesity - correlation with body mass index. Peptides 2013; 39: $125-130$.

26. Irrcher I, Adhihetty P, Sheehan T, Joseph AM, Hood A. PPAR $\gamma$ coactivator-1 $\alpha$ expression during thyroid hormone- and contractile activity-induced mitochondrial adaptations. Am J Physiol Cell Physiol 2003; 284: 1669-1677.

27. Gouni-Berthold I, Berthold HK, Huh JY, Berman R, Spenrath N, Krone W, Mantzoros CS. Effects of lipid-lowering drugs on irisin in human subjects in vivo and in human skeletal muscle cells ex vivo. PLoS One 2013; 8: 1-10.

28. Ruchala M, Zybek A, Szczepanek-Parulska E. Serum irisin levels and thyroid function-newly discovered association. Peptides 2014; 60: 51-55. 